\title{
Improving illiterate patients understanding and adherence to discharge medications
}

\author{
Matthew Clayton - Services Hospital, Lahore, Pakistan, Faizan Syed, Amjid Rashid, Umer Fayyaz
}

\begin{abstract}
Adherence to a hospital discharge medication regime is crucial for successful treatment and to avoid increasing rates of drug resistance. A patient's success in adhering to their medication regime is dependent on many social, cultural, economic, illness and therapy-related factors, and these are often more pronounced in the developing world. Anecdotal evidence in Services Hospital, Lahore (Pakistan) suggested that the relatively high levels of illiteracy in the patient population was a major factor in poor adherence. Baseline measurement revealed that $48 \%$ of all the hospital's patients were illiterate with just $5 \%-12 \%$ of illiterate patients being able to interpret their handwritten discharge prescription after leaving hospital. Unsurprisingly follow-up clinics reported very poor adherence. This quality improvement project intervened by designing a new discharge prescription proforma which used pictures and symbols rather than words to convey the necessary information. Repeated surveys demonstrated large relative increases in comprehension of the new proformas amongst illiterate patients with between $23 \%-35 \%$ of illiterate patients understanding the new proformas.
\end{abstract}

\section{Problem}

Doctors working in follow-up outpatient clinics at Services Hospital, Lahore (Pakistan) have noted very poor levels of adherence to patient's TTO ('to take home') discharge medication regime given on previous admissions and clinics. The problem is particularly marked among the large illiterate patient population served by this hospital.

This non-adherence can have serious implications for the patient's overall health outcome, with incorrectly taken medications potentially resulting in relapses of the patient's condition, medication dependence, drug resistance and potential accidental overdosing.

\section{Background}

Adherence is a concept defined by the World Health Organisation (WHO) as 'the extent to which a person's behaviour in taking medication ... corresponds with agreed recommendations from a healthcare provider' (1). The consequences of non-adherence are potentially serious, ranging from failure to treat the patient's condition and drug resistance from under-dosing, to possible fatal toxicity if overdosing takes place.

The WHO has identified five 'dimensions' of a patient's experience that can influence their adherence to medication regimes (1). These five 'dimensions' are -

(i) their social/economic circumstances (including health literacy)

(ii) factors relating the healthcare system itself (including information given out)

(iii) the nature of the patient's underlying condition (lack of symptoms or mental health issues)

(iv) factors relating to the therapy being prescribed (side effects, duration of therapy)

(v) the physical and psychological factors relating to the patient themselves (for example, individual motivation or debilitating comorbidities)

The WHO estimates that there is around $50 \%$ non-adherence to treatment regimes for chronic conditions in the developed world (1) but that this figure is likely to be even worse in the developing world where factors from each of the above five 'dimensions' of nonadherence are likely to be exacerbated.

The situation in Services Hospital, Lahore is illustrative of this. It is a large 1096 bed teaching hospital serving both a large local urban population and a rural population who attend from their villages many days travel away. The majority of patients are economically impoverished and many have very little formal education, and consequently health literacy and overall literacy levels are extremely low.

In terms of primary healthcare there is only the most rudimentary general practitioner systems outside of secondary care hospitals. For the majority of patients their only access to healthcare advice is within the walls of the hospital itself. This makes patients' understanding of their TTO ('to take out') discharge medication regimes crucial at the point of discharge as they have virtually no way of correcting any misunderstandings in terms of what they should be taking before they return for outpatient follow-up often several months down the line.

Currently, upon discharge a patient is handed a handwritten TTO discharge prescription on a loose piece of paper (and most commonly in English) by the junior doctor on the ward and told to go to the hospital pharmacy to collect their medicines. A typical prescription might use abbreviations such as 'Clarithromycin $500 \mathrm{mg}$ bd po $7 / 7$ '. A busy junior doctor may or may not have time to sit 
down and try to explain the discharge prescription to the patient.

Then at one of the extremely busy hospital pharmacies a box or unlabelled strip of tablets are given to the patient without explanation of what the medicines are or how they are to take them. Patients then return to their homes, often rural villages with no healthcare professionals within hundreds of miles, with nothing other than the boxes of medications and the doctor's handwritten TTO discharge prescription.

This is an extremely challenging situation for any patient to be in, and illiterate patients (who make up a very large percentage of the patient population) are forced to rely almost entirely on any verbal instructions they might have been given from the junior doctor on the ward. Some patients who are regular attenders have learnt to recognise the shape of certain medicine names on the prescription, whilst others receive some help back in their village from literate relatives or community members.

Unsurprisingly outpatient clinic doctors report very poor levels of adherence on the patient's subsequent return to the hospital.

\section{Baseline}

For this improvement project we first wanted to establish the percentage of the hospital's patients who were illiterate and therefore would have difficulties in interpreting their TTO discharge medication prescriptions. We then wanted to establish four outcome measures -

(i) the percentage of literate patients who could understand their TTO after an explanation from the junior doctor ('counselling') (ii) the percentage of literate who could understand their TTO without any counselling from the junior doctor

(iii) the percentage of illiterate patients who could understand their TTO with counselling from the junior doctor

(iv) the percentage of illiterate patients who could understand their TTO without any counselling from the junior doctor.

We did this by selecting a random sample of 100 medical inpatients at Services Hospital and asked each of them to read a simple sentence written in Urdu (their first language) to establish whether or not they were literate. We found that overall $52 \%$ of patients were literate and $48 \%$ of patients were illiterate.

We then showed each of the patients a typical example of a TTO discharge medication prescription. Through random selection, some patients received counselling as to what the TTO showed, whereas other patients were simply asked to interpret it themselves, thus reflecting the realities of the current situation as much as possible. The numbers of patients who were given counselling or otherwise are shown in Table 1. Each patient was then asked to explain back to the doctor the meaning of the TTO discharge prescription in terms of which drugs were prescribed, the dosages, what times of day they were to be taken, and the length of the course of treatment. without any counselling and this figure rose to $100 \%$ of literate patients understanding the TTO when given counselling with it. Amongst illiterate patients only $5 \%$ understood the TTO without any counselling, and even with counselling only $12 \%$ of illiterate patients understood the TTO correctly.

Extrapolating these percentages gives us a figure that $51 \%$ of the total patient population understand the TTO without counselling with this figure increasing to $58 \%$ of the total patient population understanding the TTO when counselling is given. Counselling the patient would therefore seem to be a worthwhile improvement.

See supplementary file: Baseline measurement.docx

\section{Design}

Instead, the agreed intervention was to design a standardised TTO discharge medication prescription for the doctor to fill in and give to the patient to take home with them. This standardised form would be picture rather than word based. For example, instructions such as 'bd' and 'tds' could be replaced by pictures of a sun rising over the mountains to represent morning, and a moon and stars to represent night time.

Along with writing the names of medications on the form each medication could also be assigned an Arabic number (which the illiterate patients were able to read). Pharmacists could then be asked to write the equivalent number on the strip of tablets or box of tablets that they dispensed to the patient.

That way, a patient would only have to recognise the number on the box of tablets rather than read the name of the medication. Posters could be produced to educate the pharmacists on this simple intervention. This intervention was felt to be effective for the following -

(i) it would inexpensive to print out black and white standardised TTO discharge medication proformas in bulk

(ii) the use of pictures rather than words on the form was more likely to make the form comprehensible to illiterate patients who could then keep the form as a reference point of the correct dosages/timings/length of treatment throughout their course of treatment

(iii) the form could be easily edited should changes become desirable at a later date.

(iv) the new form would not take any more time than the old system for the busy junior doctors

(v) the new system involves minimal co-operation from the pharmacists apart from writing the corresponding number for each medication from the form onto the box of tablets they are dispensing

The new standardised TTO discharge medication proforma was designed and following various revisions the final form was trialed among the same 100 medical inpatients previously shown a handwritten TTO. 


\section{Strategy}

PDSA Cycle 1

An example of a TTO discharge medication regime using the new picture-based proforma was shown to a selection of illiterate patients and junior doctors involved in the discharge process in order to get their feedback. Whilst the illiterate patients liked and understood the use of the image of a sun rising and setting over the mountains to represent various times of day and night they were confused by the use of a calendar to represent the number of days that the treatment was to run for.

A hospital pharmacist was shown the new discharge form and asked to label the medication boxes appropriately. He found the form easy to follow and understand.

PDSA Cycle 2

The form was redesigned using the symbol of the sun, rather than a calendar, to represent the number of days of treatment. Our illiterate patient sample group decided that whilst still not perfect, it was big improvement on the calendar, and that it was far more intuitively understood.

We trialed the new proforma with a larger group of pharmacists this time asking them to correctly complete the labelling using only our educational poster as a guide. They were all able to do this without difficulty.

See supplementary file: userfiles-Discharge PDSA cycles.pdf

\section{Post-Measurement}

We wished to measure the impact of the new TTO, so we showed each of the 100 medical patients used in the baseline measurement a sample of a new picture-based discharge regime and asked them to explain back to us the discharge medications, the dosages, the time of day they should take them, and the length of the course of treatment on that pro-forma. Patients were randomly selected to receive counselling. The results were as follows -

- Literate patients who received counselling with the new proforma understood it $100 \%$ of the time (vs. $100 \%$ with the old handwritten TTO)

- Literate patients who did not receive counselling with the new proforma understood it $100 \%$ of the time (vs. $93 \%$ with the old handwritten TTO)

- Illiterate patients who received counselling with the new proforma understood it $35 \%$ of the time (vs. $12 \%$ with the old handwritten TTO)

- Illiterate patients who did not receive counselling with the new proforma understood it $23 \%$ of the time (vs. $5 \%$ with the old handwritten TTO)
The new picture-based discharge proforma has markedly improved understanding of discharge medication regimes among illiterate patients. We demonstrated a relative increase in understanding of $192 \%$ among illiterate patients who received counselling and a relative increase of $360 \%$ among illiterate patients who were not counselled.

See supplementary file: userfiles-Supporting_documents.pdf

\section{Lessons and Limitations}

1) Improving patient care is possible in a short space of time with hard work and motivation. Introducing the first new picture-based discharge proformas took less than a week with a team of four junior doctors.

2) Engaging stakeholders is key in any quality improvement project. We knew that the senior staff would ultimately decide whether or not the proformas would be allowed to be used on their wards. Presenting the project to them together with evidence from repeated patient surveys allowed us to persuade and demonstrate the benefits of the pro forma winning their approval.

3) Carrying out PDSA cycles as part of the design of the final proforma was a crucial step in increasing illiterate patients understanding of the discharge. Without these, a major flaw in the original design - that the symbol of a calendar was very poorly understood especially by rural villagers - would not have been picked up upon and subsequently redesigned.

\section{Conclusion}

The problem that we started with was that illiterate patients, who made up around half of the entire patient population, were being poorly served by the old discharge system. Illiterate patients were simply being handed a loose piece of paper containing a handwritten prescription by busy junior doctors. Illiterate patients were not only unable to read the names of the medication on the boxes but also were confounded by the handwritten prescription instructing them how to take their medicines.

The picture-based discharge medication proforma which was developed along with hospital pharmacists numbering the boxes of medication was designed to combat this problem. The patient surveys seem to show success in this area with $23 \%-35 \%$ of illiterate patients understanding the new proforma (depending on the level of counselling given) versus only $5 \%-12 \%$ of illiterate patients understanding the old handwritten TTOs.

The new proforma increased the number of illiterate patients who can understand their discharge medication threefold but it is important to recognise that there is still room for improvement. Even using the new proforma the majority of illiterate patients can still not understand the new system.

The proforma will benefit from further reviews and by using patient 


\section{BMJ Quality Improvement Reports}

feedback to maximise illiterate patients' comprehension of it as much as possible. For example, many patients found the use of a picture of the sun to represent the number of days of a course of treatment not immediately intuitive. We feel future improvements in the design of the form could be made in areas such as this.

Secondly if the ultimate aim of the project was to improve patients' adherence to their medication regimes then we should remember that this project does not actually directly measure compliance at any stage. Whilst it seems reasonable that having a prescription that is better understood by vast illiterate patient population will improve overall adherence this is an assumption rather than evidence-based at present.

Such a measurement would of course be fraught with difficulty due to the numerous influences on adherence. It is for similar reasons that we were unable to calculate the total resource savings for this project.

Despite these difficulties it will be interesting to gather anecdotal evidence on the success of this project from doctors and patients in the future. We feel the innovation is likely to be of benefit to large numbers of patients, particularly in the illiterate population, and as such feel the project is applicable to other patient populations with low literacy rates elsewhere in the developing world.

\section{References}

Adherence to long-term therapies: evidence for action. World Health Organization, 2003. Available at:

http://www.who.int/chronic_conditions/adherencereport/en/ 